Braz J Med Biol Res, September 2012, Volume 45(9) 799-805

doi: 10.1590/S0100-879X2012007500114

Correlation between sodium and potassium excretion in 24- and 12-h urine samples

J.G. Mill, A.B.T. da Silva, M.P. Baldo, M.C.B. Molina and S.L. Rodrigues

The Brazilian Journal of Medical and Biological Research is partially financed by

\section{Q}

da Ciência e Tecnologia

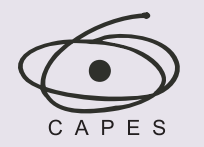

Ministério da Educação
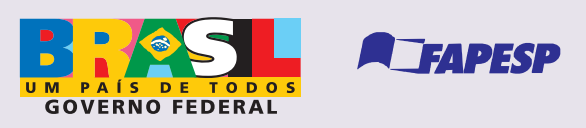

Institutional Sponsors

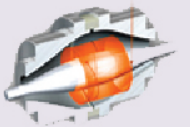

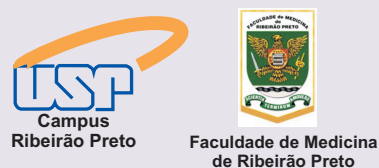

de Ribeirão Preto
de

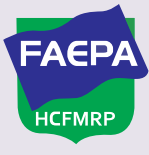

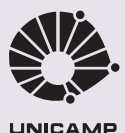

UNICAMP
Ф SHIMADZU

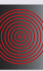

A디맘

Associaçäo
Fundo
del Incentivo de lincentivo
à Pesquisa

plore High - Performance MS Orbitrap Technology andilicica Thermo 


\title{
Correlation between sodium and potassium excretion in 24- and 12-h urine samples
}

\author{
J.G. Mill ${ }^{1}$, A.B.T. da Silva ${ }^{1}$, M.P. Baldo ${ }^{1}$, M.C.B. Molina ${ }^{2}$ and S.L. Rodrigues ${ }^{1}$ \\ ${ }^{1}$ Departamento de Ciências Fisiológicas, Universidade Federal do Espírito Santo, Vitória, ES, Brasil \\ 2Departamento de Ciências da Saúde, Universidade Federal do Espírito Santo, Vitória, ES, Brasil
}

\begin{abstract}
Low-sodium and high-potassium diets have been recommended as an adjunct to prevention and treatment of hypertension. Analysis of these nutrients in 24-h urine has been considered the reference method to estimate daily intake of these minerals. However, 24-h urine collection is difficult in epidemiological studies, since urine must be collected and stored in job environments. Therefore, strategies for shorter durations of urine collection at home have been proposed. We have previously reported that collecting urine during a 12-h period (overnight) is more feasible and that creatinine clearance correlated strongly with that detected in 24-h samples. In the present study, we collected urine for $24 \mathrm{~h}$ divided into two 12-h periods (from 7:00 am to 7:00 $\mathrm{pm}$ and from 7:00 pm to 7:00 am next day). A sample of 109 apparently healthy volunteers aged 30 to 74 years of both genders working in a University institution was investigated. Subjects with previous myocardial infarction, stroke, renal insufficiency, and pregnant women were not included. Significant $(P<0.001)$ Spearman correlation coefficients $\left(r_{s}\right)$ were found between the total amount of sodium and potassium excreted in the urine collected at night and in the 24-h period $\left(r_{s}=0.76\right.$ and 0.74 , respectively). Additionally, the 12-h sodium and potassium excretions (means \pm SD, $95 \%$ confidence interval) corresponded to $47.3 \pm 11.2 \%, 95 \% \mathrm{Cl}=45.3-49.3$, and $39.3 \pm 4.6 \%, 95 \% \mathrm{Cl}=37.3-41.3$, respectively, of the $24-\mathrm{h}$ excretion of these ions. Therefore, these findings support the assumption that 12-h urine collected at night can be used as a reliable tool to estimate 24-h intake/excretion of sodium and potassium.
\end{abstract}

Key words: Sodium excretion; Urine; Salt intake; Potassium excretion

\section{Introduction}

High-salt diets have been associated with a greater risk of hypertension, stroke, and other cardiovascular diseases (1). Data from epidemiological and experimental studies have consistently demonstrated the blood pressurelowering effect of reduced salt intake by hypertensive subjects (2-4). In contrast, high potassium intake has been associated with blood pressure lowering (5). Therefore, diets with reduced sodium and increased potassium intake have been recommended for hypertensive individuals as a non-pharmacological strategy for blood pressure control. Moreover, adoption of such healthy diet may also prevent the future development of hypertension in normotensive individuals (6).

Several methods have been used to estimate sodium and potassium intake. In general, this information has been obtained by applying questionnaires or by measuring urine excretion of these ions. However, the use of dietary recall to estimate nutritional salt intake and the analysis of duplicate diets have been considered unsatisfactory research tools (7-9) due to imprecise self-reporting of food intake or inaccurate and incomplete food databases (10). Thus, analysis of sodium and potassium content in urine collected over $24 \mathrm{~h}$ has been long considered the reference method to estimate the daily intake of these minerals (11). However, the difficulty in collecting all urine produced over $24 \mathrm{~h}$ is an important limitation, particularly in epidemiologic studies involving active working subjects. Under such conditions, the rate of unsuccessful collection has been as high as $40 \%$ of subjects (12).

Due to this lack of success, other strategies for urine collection have been proposed. We have previously reported that collecting urine during a 12-h period (overnight) is more feasible (13) than collecting urine over a 24 -h period and that the 12-h creatinine clearance correlated strongly with that observed during the 24-h period (14). Our group is currently involved in a large prospective cohort study of

Correspondence: J.G. Mill, Departamento de Ciências Fisiológicas, Centro de Ciências da Saúde, Universidade Federal do Espírito Santo, Av. Marechal Campos, 1468, 29042-755 Vitória, ES, Brasil. Fax: +55-27-3335-7330. E-mail: jgmill@npd.ufes.br

Received November 16, 2011. Accepted June 22, 2012. Available online July 13, 2012. Published August 17, 2012. 
Brazilian adults (Estudo Longitudinal de Saúde do Adulto, ELSA-Brazil Project) in which kidney function and diet habits are being investigated (15). In the present study, these parameters were evaluated by overnight 12-h urine collection. The objective of the study was to determine the correlation between sodium and potassium excreted during a 12-h period (night) and the gold standard, i.e., 24-h urine collection. In addition to the shorter and more convenient data collection period, night collection can reduce the biases associated with salt loss from sweat during daily activities and/or hot weather, resulting in higher diagnostic accuracy.

\section{Material and Methods}

\section{Study design and population}

A subsample of participants of the ELSA-Brazil cohort and workers from the University Hospital were invited to participate in this cross-sectional observational study. A total of 115 subjects of both genders aged 30 to 74 years were invited and agreed to participate. The University Hospital conducted clinical and laboratory exams to evaluate the presence of cardiovascular risk factors. Pregnant women and individuals with self-reported heart and kidney diseases were excluded. Participants were instructed to avoid strenuous exercise and alcohol consumption for $24 \mathrm{~h}$ preceding the clinical visit. The project was approved by the Institutional Ethics Committee (UFES) and all subjects gave informed written consent to participate in the study.

\section{Exams}

Blood pressure (BP) was measured according to standard procedures in the morning (between 7:00 and 9:00 am) after a 12-h fast. The participants were asked to empty their bladders and BP was measured on the left arm after a rest period of 5-10 min in the sitting position. Three measurements were obtained at 5-min intervals. Mean values of systolic and diastolic BP were calculated from the mean of the last two measurements. A validated and automated oscillometric device (Omron 765CP, Japan) was used. Subjects were considered to be hypertensive in the presence of systolic BP $\geq 140 \mathrm{mmHg}$ or diastolic BP $\geq 90 \mathrm{mmHg}$ or if they were using anti-hypertensive drugs, including diuretics. Anthropometric parameters were obtained by trained technicians using standard methods. Body weight was measured to the nearest $0.1 \mathrm{~kg}$ on a calibrated scale. Height was measured to the nearest 0.5 $\mathrm{cm}$ with a wall-mounted stadiometer. Body mass index (BMI) was calculated as body weight in kilograms divided by height in meters squared $\left(\mathrm{kg} / \mathrm{m}^{2}\right)$. Waist circumference was measured at the mean point between the lowest rib margin and iliac crest while the subject was standing and at the maximum point of normal expiration.

Fasting blood samples were collected soon after BP measurements to determine glucose, triglycerides, total cholesterol, HDL cholesterol lipoprotein, and uric acid. Diabetes was defined as a positive history, use of antidiabetic drugs, or a fasting blood glucose $>125 \mathrm{mg} / \mathrm{dL}$. All biochemical analyses were performed using validated commercial analytic kits in a single reference laboratory.

\section{Urine collection}

The research staff previously met with the participants to give instructions regarding urine collection and specimen handling. The urine collection kit comprised two labeled (day and night) sterile plastic containers ( $2 \mathrm{~L}$ ) and detailed written instructions. Urine was collected during the 24 -h period preceding the clinic visit. Therefore, urine was collected during weekdays or Sundays. The 24-h urine collection was divided into two time periods: the day period (from 7:00 am to 7:00 pm) and the night period (from 7:00 pm to 7:00 am). When the containers were not in use, the staff instructed the participants to keep them in the refrigerator. The importance of separating the two 12-h collection periods into two separate bottles was strongly emphasized. Each participant was asked to record the time of last voiding in the toilet before starting urine collection. They were also instructed to record the beginning and ending time of each specimen collection and if some urine was lost during the collection period. On returning the $24-\mathrm{h}$ urine sample, the participants' annotations were analyzed and participants were thoroughly questioned about completeness. The threshold for validity of the urine specimen as representative of a 24-h period was a volume greater than $500 \mathrm{~mL} /$ day (at least $250 \mathrm{~mL} /$ each 12-h period). Six participants were excluded from the analysis because of an inappropriately low volume of urine collection. Sodium and potassium concentrations in the urine specimens were measured using calibrated selective electrode devices.

\section{Statistical analysis}

Statistical analysis was conducted using the SPSS 18.0 statistical package (USA). Data are reported as means \pm SD and $95 \%$ confidence intervals $(95 \% \mathrm{Cl})$. Proportions were used to express dichotomous variables. The goodness of fit for normal distribution was evaluated using the Kolmogorov-Smirnov test. To test for differences in the means across groups, the unpaired or paired $t$-test was used for continuous variables and the chi-square test was used for categorical variables. The association between urinary parameters in 12- and 24-h urine samples was determined using the Pearson ( $r$ ) or Spearman $\left(r_{s}\right)$ correlation coefficient as appropriate. BMI and triglycerides were analyzed after log transformation. Statistical significance was set at $P<0.05$.

\section{Results}

A total of 109 participants were included in the analysis. The general characteristics of the sample stratified 
by gender are shown in Table 1. The prevalence of hypertension, diabetes, smoking, obesity, and diuretic use was $37.6,7.3,12.8,24.1$, and $13.8 \%$, respectively, with no difference between genders. Systolic BP, weight, and plasma triglycerides and creatinine were higher in men, whereas heart rate and HDL cholesterol were higher in women. Age, diastolic BP, BMI, total cholesterol, and serum glucose were similar for the two genders.

The general characteristics of 24-h urine collected during the day and night periods are shown in Table 2. Urine volume and flow were similar in men and women, with no significant difference between the collection periods. Mean sodium excretion was $4.72 \mathrm{~g}$ over 24 $\mathrm{h}$, corresponding to approximately $12 \mathrm{~g}$ $\mathrm{NaCl}$ per day. Sodium elimination during the night comprised $47.3 \pm 11.2 \%$ $(95 \% \mathrm{Cl}=45.3-49.3)$ of the $24-\mathrm{h}$ excretion. The $24-h$ creatinine excretion was higher in men than in women (1484 \pm 374 vs $1002 \pm 282$ mg; $P<0.05)$ both during the day and night periods (data not shown). Urinary creatinine adjusted for body weight remained higher in men (19.3 \pm 4.1 vs $14.6 \pm 3.2 \mathrm{mg} / \mathrm{kg} ; \mathrm{P}<$ $0.01)$. Creatinine excretion during the night period was equivalent to $48.5 \pm$ $4.7 \%(95 \% \mathrm{Cl}=46.5-51.5)$ of the total 24-h excretion, a relative value close to the nocturnal sodium excretion. As expected, the difference in sodium excretion between the nocturnal and diurnal periods disappeared when sodium was corrected for creatinine.

Urine collected during the day and night periods showed different characteristics when potassium excretion was analyzed. Excretion of this ion was significantly lower during the night period, comprising $39.3 \pm 4.6 \%$ of the total $24-\mathrm{h}$ excretion $(95 \% \mathrm{Cl}=37.3-41.3$ of the $24-\mathrm{h}$ urine). The difference between the night and day periods remained statistically significant even after adjustment for urinary creatinine excretion.

Correlations between crude sodium and potassium excretion during the night and the 24-h period are shown Figure 1. A strong Spearman correlation coefficient $\left(r_{s}=0.76, P\right.$ $<0.001$ ) between nocturnal and 24-h sodium excretion was observed. This correlation remained strong even after stratification by gender $\left(r_{S}=0.77, P<0.001 ; r_{S}=0.74, P<\right.$ periods. $t$-test).
Table 1. General characteristics of the sample stratified by gender.

\begin{tabular}{lccc}
\hline & All $(\mathrm{N}=109)$ & Men $(\mathrm{N}=50)$ & Women (N = 59) \\
\hline Age (years) & $50.7 \pm 10.4$ & $50.9 \pm 10.4$ & $50.7 \pm 10.5$ \\
Heart rate (bpm) & $70 \pm 10$ & $67 \pm 10$ & $71 \pm 9^{*}$ \\
Systolic blood pressure (mmHg) & $121 \pm 17$ & $125 \pm 18$ & $117 \pm 14^{*}$ \\
Diastolic blood pressure (mmHg) & $78 \pm 10$ & $79 \pm 12$ & $76 \pm 8.0$ \\
Body mass index (kg/m²) & $27.3 \pm 4.2$ & $26.8 \pm 3.6$ & $27.6 \pm 4.6$ \\
Weight (kg) & $72.3 \pm 12.2$ & $76.8 \pm 10.4$ & $68.6 \pm 12.1^{*}$ \\
Cholesterol (mg/dL) & $203.4 \pm 46.1$ & $197.1 \pm 50.7$ & $208.7 \pm 41.6$ \\
Triglycerides (mg/dL) & $135.8 \pm 108.1$ & $156.3 \pm 119.8$ & $118.4 \pm 94.6$ \\
HDL cholesterol (mg/dL) & $46.1 \pm 11.5$ & $43.3 \pm 12.5$ & $48.5 \pm 11.1^{*}$ \\
Glucose (mg/dL) & $100.7 \pm 29.6$ & $106.1 \pm 38.3$ & $96.1 \pm 18.5$ \\
Creatinine (mg/dL) & $0.95 \pm 0.14$ & $1.0 \pm 0.1$ & $0.86 \pm 0.11^{*}$ \\
Hypertension (N, \%) & $41(37.6)$ & $20(40)$ & $21(35.6)$ \\
Diabetes mellitus (N, \%) & $8(7.3)$ & $5(10)$ & $3(5.1)$ \\
Smoking (N, \%) & $26(12.8)$ & $21(18)$ & $5(8.5)$ \\
Obesity (N, \%) & $26(24.1)$ & $9(18.4)$ & $17(28.8)$ \\
Diuretic use (N, \%) & $15(13.8)$ & $6(12)$ & $9(15.3)$ \\
\hline
\end{tabular}

Data are reported as means $\pm S D$, unless otherwise indicated. ${ }^{*} P \leq 0.05$ compared to men (unpaired $t$-test for continuous variables and $\mathrm{x}^{2}$ for categorical variables).

Table 2. Characteristics of the urine collected during 24-h, 12-h day and 12-h night

\begin{tabular}{lccc}
\hline & $24 \mathrm{~h}$ & Day & Night \\
\hline Volume $(\mathrm{L})$ & $2.12 \pm 0.94$ & $1.04 \pm 0.55$ & $1.08 \pm 0.49$ \\
Flow $(\mathrm{mL} / \mathrm{min})$ & $1.47 \pm 0.65$ & $1.44 \pm 0.76$ & $1.49 \pm 0.67$ \\
Creatinine $(\mathrm{mg})$ & $1223 \pm 405$ & $630 \pm 232$ & $593 \pm 214^{*}$ \\
Creatinine $(\mathrm{mg} / \mathrm{dL})$ & $70.8 \pm 42.8$ & $78.5 \pm 50.6$ & $68.7 \pm 43.4^{*}$ \\
$\mathrm{Na}(\mathrm{mEq} / \mathrm{L})$ & $110 \pm 49$ & $122 \pm 54$ & $104 \pm 53^{*}$ \\
$\mathrm{Na}(\mathrm{mg})$ & $4721 \pm 1623$ & $2488 \pm 992$ & $2233 \pm 962^{*}$ \\
$\mathrm{Na} / \mathrm{creatinine}(\mathrm{mg} / \mathrm{mg})$ & $3.9 \pm 1.5$ & $3.9 \pm 1.8$ & $3.7 \pm 1.9$ \\
$\mathrm{~K}(\mathrm{mEq} / \mathrm{L})$ & $31 \pm 14$ & $41 \pm 20$ & $25 \pm 14^{*}$ \\
$\mathrm{~K}(\mathrm{mg})$ & $2228 \pm 710$ & $1351 \pm 476$ & $876 \pm 387^{*}$ \\
$\mathrm{~K} / \mathrm{creatinine}(\mathrm{mg} / \mathrm{mg})$ & $1.8 \pm 0.7$ & $2.1 \pm 1.0$ & $1.5 \pm 0.6^{*}$ \\
$\mathrm{Na} / \mathrm{K}(\mathrm{mEq} / \mathrm{mEq})$ & $3.8 \pm 1.6$ & $3.3 \pm 1.3$ & $4.8 \pm 2.6^{*}$ \\
\hline
\end{tabular}

Data are reported as means $\pm S D$. ${ }^{*} \mathrm{P} \leq 0.05$ compared to day collection (unpaired

0.001 , for men and women, respectively). When analyzed according to diuretic use, the correlation was still significant $(\mathrm{P}<0.001)$ in the subgroup without diuretics $\left(\mathrm{N}=94 ; r_{\mathrm{S}}=\right.$ $0.82)$. Significance was lost in the subgroup under diuretics use $\left(r_{\mathrm{s}}=0.44 ; P=0.09\right)$. The small number of subjects in this subgroup $(\mathrm{N}=15)$ should have contributed to this finding. Assuming linearity between the two measures, 24-h sodium excretion $(\mathrm{mg})$ can be estimated by the equation: $\mathrm{Na}_{24} \mathrm{~h}(\mathrm{mg})=1614.1+1.39 \times \mathrm{Na}_{12}$-h night $(\mathrm{mg})$.

The $95 \% \mathrm{Cl}$ was $1169.9-2058.4$ for the linear coefficient 

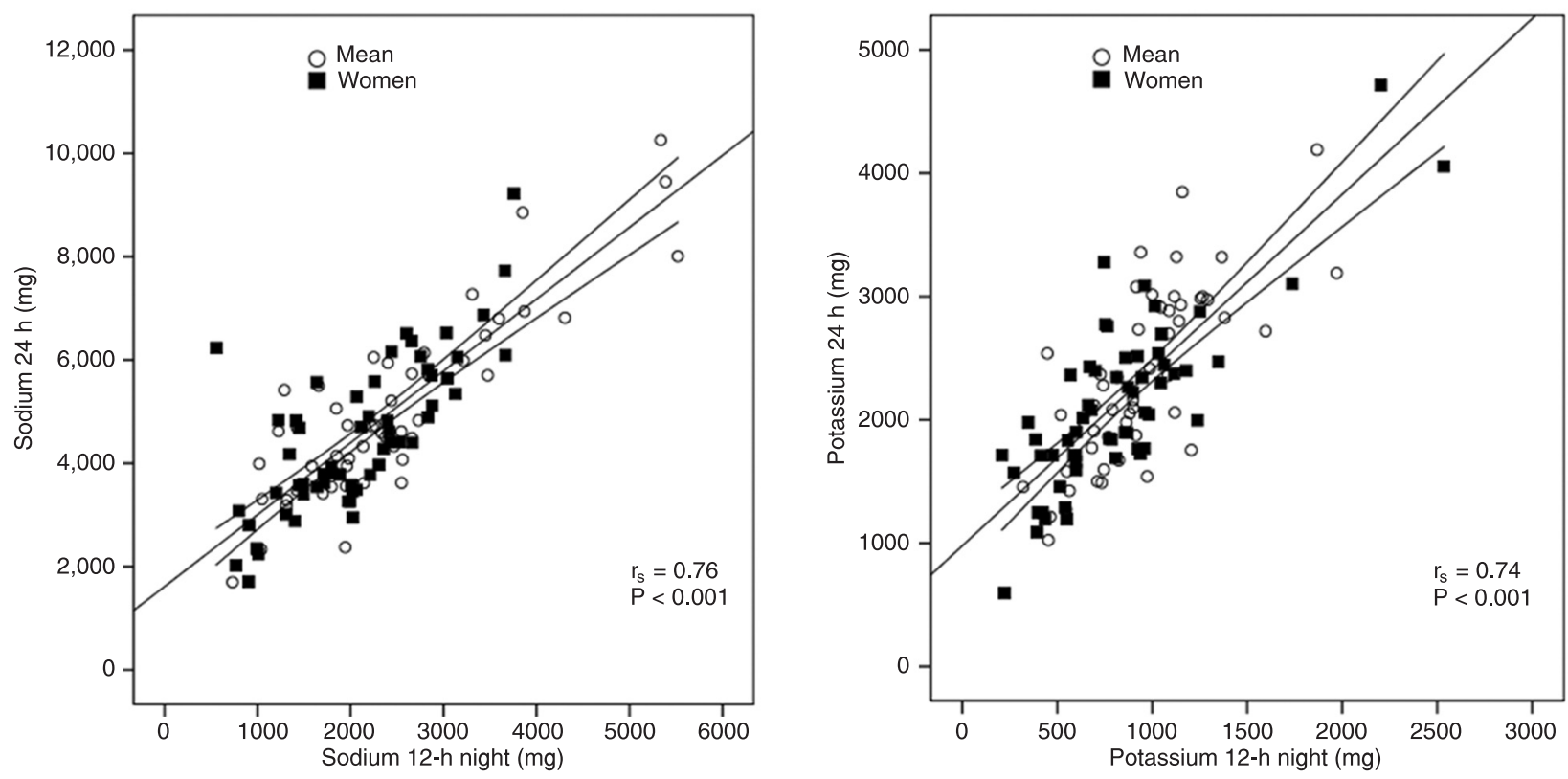

Figure 1. Correlation between sodium and potassium measured in urine collected during the night and during a 24-h period. The Figure shows the regression line and the $95 \%$ confidence interval of the regression. $r_{\mathrm{s}}=$ Spearman correlation coefficient.

and $1.20-1.57$ for the angular coefficient.

The correlation between 12-h nocturnal and 24-h potassium excretion is shown in Figure 1. The graph also shows a strong association in the total sample $\left(r_{s}=0.74\right.$, $P<0.001$ ), which remained strong even after stratification for gender $\left(r_{s}=0.75, P<0.001 ; r_{S}=0.73, P<0.001\right.$, for men and women, respectively). The correlation coefficient for the 12-h nocturnal and 24-h potassium excretion was high in the subgroups under and without diuretics $\left(r_{s}=0.80\right.$ and 0.75 , respectively). Assuming linearity between the two measures, the 24-h potassium excretion (in $\mathrm{mg}$ ) can be estimated by the equation: $\mathrm{K}_{24 \mathrm{~h}(\mathrm{mg})}=978.9+1.42 \mathrm{x}$

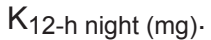

The $95 \% \mathrm{Cl}$ was $766.9-1190.6$ for the linear coefficient and 1.20-1.64 for the angular coefficient.

Sodium and potassium excretion over the 12-h night and 24-h periods maintained a significant linear correlation even after adjustment for creatinine excretion ( $\mathrm{r} \mathrm{Na} /$ creatinine $=$ $0.84 ; \mathrm{P}<0.001$ and $\mathrm{K}$ K/creatinine $=0.75 ; \mathrm{P}<0.001$; Figure 2). A lower association was observed in women, mainly for potassium excretion, which can be explained by the lower creatinine excretion in women.

\section{Discussion}

Consumption of several nutrients, including minerals, has an important influence on health status and the development of several chronic diseases $(5,6,11)$. In a recent meta-analysis of 31 trials, an average decrease of 5.0 $\mathrm{mmHg}$ for systolic BP and $2.7 \mathrm{mmHg}$ for diastolic BP was detected in hypertensive patients after reduction of sodium consumption by $75 \mathrm{mmol} /$ day (16), equivalent to $4 \mathrm{~g}$ salt per day. The prevalence of hypertension is approximately 25 to $30 \%$ of the adult Brazilian population (17). The association between salt consumption and mean blood pressure levels and the prevalence of hypertension has been well established in the literature $(16,18,19)$. Therefore, reduction of sodium ingestion may significantly decrease BP levels and the prevalence of hypertension in our population because salt consumption has been shown to be high, exceeding 12 g/day, mainly in hypertensive subjects (13). An adequate evaluation of salt consumption is a fundamental tool to establish public health policies for target populations.

Most studies estimate sodium and potassium intake with analytic measurements of food consumed, food surveys, or dietary records $(20,21)$. However, most of these methods have failed to be reliable indicators of nutrient consumption because the quantities of food consumed are reported inadequately by subjects and several instruments used to calculate food composition do not cover regional characteristics of food preparations (7-10). Therefore, urine collection is more reliable than other methods to assess sodium and potassium consumption, as detailed studies in humans have confirmed that approximately $90 \%$ of sodium consumed and $77 \%$ of potassium consumed are eliminated in urine collected along $24 \mathrm{~h} \mathrm{(22).}$

Urinary excretion over a $24-\mathrm{h}$ period has been used as the gold standard to establish patterns of sodium and potassium consumption (11). However, the major limitation of this method is the inaccuracy of the urine volume. 

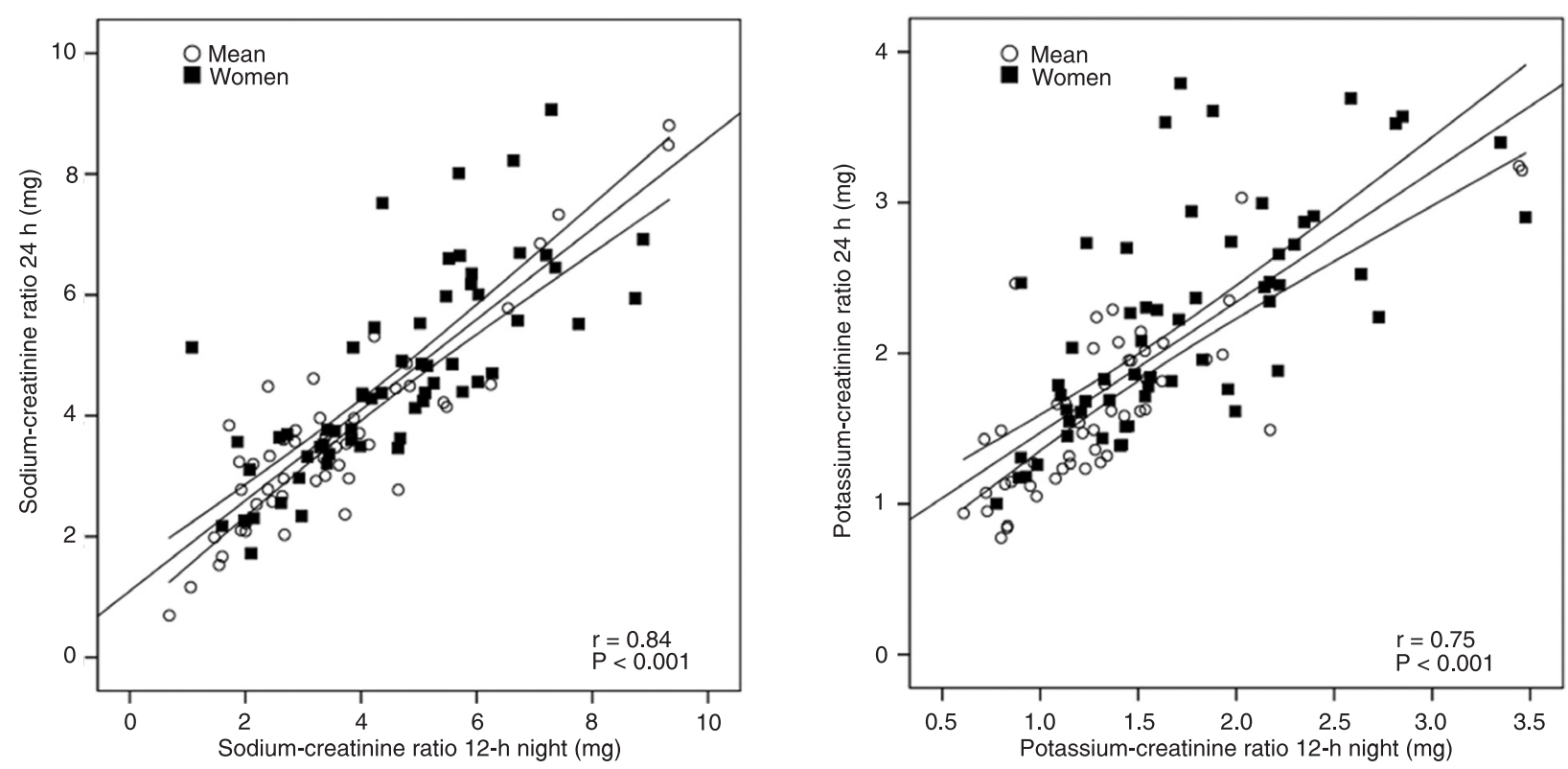

Figure 2. Correlation between sodium-creatinine ratio and potassium-creatinine ratio calculated with urine collected during the night and during a 24-h period. The Figure shows the regression line and the $95 \%$ confidence interval of the regression. $r=$ Pearson correlation coefficient.

Therefore, urine collection during shorter periods of time may be more adequate for patients and determine more accurate estimates of food intake. However, the occurrence of circadian variations secondary to urine filtration and tubular secretion or reabsorption may introduce other estimate deviations besides those secondary to urine collection (23). In large epidemiological studies conducted on the general population, measuring urine produced over 24 $h$ is challenging because specimens must be collected and stored during working periods. This collecting period may increase errors and therefore lead to inaccurate estimates. Some studies have tried to circumvent these difficulties by analyzing the sodium-to-creatinine ratio in a single urine sample (24). Our data confirm that while this procedure can be useful to estimate sodium intake, it may produce important errors in estimating potassium ingestion because there is an important circadian variation in urinary excretion of potassium (25). Moreover, our data suggest that the assumption of linearity in the potassium-to-creatinine ratio over different periods may be inadequate, mainly in women.

To bypass such difficulties we have proposed a 12-h nocturnal urine collection to estimate the daily ingestion of sodium and potassium (13). In a previous study (14), we have shown that the values of creatinine clearance calculated from 24- and 12-h urine samples were similar, a finding confirmed in the present study. We also observed that assumption of linearity between sodium and potassium excretions measured in urine collected at night and the urine collected over $24 \mathrm{~h}$ can be accepted within statistical pa- rameters. Our study indicates that the nocturnal sodium and potassium excretions represent 47 and $39 \%$, respectively, of the 24-h excretion of these ions. The confidence interval of nearly $2 \%$ may introduce an acceptable deviation from the true value for each individual, particularly if we consider that the day-to-day variation in sodium and potassium intake and excretion is generally higher than 10\% (26).

Our findings support the assumption that a shorter urine collection period can be used as a reliable tool to estimate the 24-h intake and excretion of sodium and potassium. Other studies, however, have observed poor consistency between the day and night periods of urine collection, mainly for potassium $(27,28)$. In those studies, however, the 24-h urine collection was not divided into two equal periods. Bankir et al. (28) studied a sample of 325 individuals of African descent with mean day and night collection periods of 14.1 and $9.2 \mathrm{~h}$, respectively. Therefore, most of the nocturnal urine was produced during the sleeping period, when the filtration rate tends to decline following the usual blood pressure dipping (23). It is noteworthy that in our study urinary flow was similar during the day and night periods. In addition, the population included in the study of Bankir et al. (28) showed a low sodium excretion (103 $\mathrm{mmol} / 24 \mathrm{~h}$ ). In a subgroup analysis of our cohort we also observed a loss of correlation between 12-h nocturnal and 24-h $\mathrm{Na}$ and $\mathrm{K}$ excretion in low sodium consumers (<100 $\mathrm{mEq} / 24 \mathrm{~h}$ ). However, even in this analysis the Pearson's $r$ coefficient was always $>0.70$ (data not shown). One limiting factor in our study is the poor estimate of the "true" sodium 
and potassium intake obtained in a single urine collection (29). However, this same limitation exists for 24- and 12-h collections. Moreover, most of the more reliable predictions of salt consumption as the general habit of a referred population have been based on 24-h urine collections. Thus, the 24-h sodium excretion can roughly be calculated in epidemiological studies as the 12-h excretion divided by 0.47 , while the $24-h$ potassium excretion can be calculated as the 12 -h excretion divided by 0.39 . Estimated errors are within $2-3 \%$, which are considered acceptable errors for estimating the intake of these minerals. However, due to

\section{References}

1. Strazzullo P, D’Elia L, Kandala NB, Cappuccio FP. Salt intake, stroke, and cardiovascular disease: meta-analysis of prospective studies. BMJ 2009; 339: b4567.

2. Dickinson HO, Mason JM, Nicolson DJ, Campbell F, Beyer FR, Cook JV, et al. Lifestyle interventions to reduce raised blood pressure: a systematic review of randomized controlled trials. J Hypertens 2006; 24: 215-233.

3. He FJ, MacGregor GA. Effect of longer-term modest salt reduction on blood pressure. Cochrane Database Syst Rev 2004; CD004937.

4. Jurgens G, Graudal NA. Effects of low sodium diet versus high sodium diet on blood pressure, renin, aldosterone, catecholamines, cholesterols, and triglyceride. Cochrane Database Syst Rev 2004; CD004022.

5. Espeland MA, Kumanyika S, Yunis C, Zheng B, Brown WM, Jackson $\mathrm{S}$, et al. Electrolyte intake and nonpharmacologic blood pressure control. Ann Epidemiol 2002; 12: 587-595.

6. Kesteloot H, Tzoulaki I, Brown IJ, Chan Q, Wijeyesekera A, Ueshima $\mathrm{H}$, et al. Relation of urinary calcium and magnesium excretion to blood pressure: The International Study of Macro- and Micro-nutrients and Blood Pressure and the International Cooperative Study on Salt, Other Factors, and Blood Pressure. Am J Epidemiol 2011; 174: 44-51.

7. Schachter J, Harper PH, Radin ME, Caggiula AW, McDonald $\mathrm{RH}$, Diven WF. Comparison of sodium and potassium intake with excretion. Hypertension 1980; 2: 695-699.

8. Hill LL, Montandon CM, Scott L, Hammond GS, Tristan MP, Baer PE. Validation of a salt intake questionnaire by urinary electrolytes excretion. Prev Med 1980; 9: 436.

9. Pietinen P, Tanskanen A, Tuomilehto J. Assessment of sodium intake by a short dietary questionnaire. Scand $J$ Soc Med 1982; 10: 105-112.

10. Espeland MA, Kumanyika S, Wilson AC, Reboussin DM, Easter L, Self M, et al. Statistical issues in analyzing 24-hour dietary recall and 24-hour urine collection data for sodium and potassium intakes. Am J Epidemiol 2001; 153: 9961006.

11. Willett W. Nutritional epidemiology. 2nd edn. New York: Oxford University Press; 1998.

12. Liu K, Dyer AR, Cooper RS, Stamler R, Stamler J. Can overnight urine replace 24-hour urine collection to asses salt intake? Hypertension 1979; 1: 529-536.

13. Molina MCB, Cunha RS, Herkenhoff FL, Mill JG. Hypertension and salt intake in an urban population. Rev Saúde the high intra-individual variability from one day to another, repeated urine collections are necessary for a precise estimation of the intake of these minerals.

\section{Acknowledgments}

Research supported by the Brazilian Ministry of Health (DECIT), FINEP (\#01.001.300.00) and CNPq (\#5619592010/2). A.B.T. da Silva and M.P. Baldo were recipients of fellowships from CAPES and CNPq, respectively.

Pública 2003; 37: 743-750.

14. Silva ABT, Molina MCB, Rodrigues SL, Pimentel EB, Baldo MP, Mill JG. Correlation between the creatinine clearance in the urine collected during 24 hours and 12 hours. J Bras Nefrol 2010; 32: 165-172.

15. Aquino EM, Barreto SM, Bensenor IM, Carvalho MS, Chor D, Duncan BB, et al. Brazilian Longitudinal Study of Adult Health (ELSA-Brasil): objectives and design. Am J Epidemiol 2012; 175: 315-324.

16. He FJ, MacGregor GA. Effect of modest salt reduction on blood pressure: a meta-analysis of randomized trials. Implications for public health. J Hum Hypertens 2002; 16: 761-770.

17. Lessa I, Mendonca GA, Teixeira MT. Non-communicable chronic diseases in Brazil: from risk factors to social impact. Bol Oficina Sanit Panam 1996; 120: 389-413.

18. Elliott P, Stamler J, Nichols R, Dyer AR, Stamler R, Kesteloot $\mathrm{H}$, et al. Intersalt revisited: further analyses of 24 hour sodium excretion and blood pressure within and across populations. Intersalt Cooperative Research Group. BMJ 1996; 312: 1249-1253.

19. Dumler F. Dietary sodium intake and arterial blood pressure. J Ren Nutr 2009; 19: 57-60.

20. Liu K, Cooper R, McKeever J, McKeever P, Byington R, Soltero I, et al. Assessment of the association between habitual salt intake and high blood pressure: methodological problems. Am J Epidemiol 1979; 110: 219-226.

21. Pietinen P. Estimating sodium intake from food consumption data. Ann Nutr Metab 1982; 26: 90-99.

22. Holbrook JT, Patterson KY, Bodner JE, Douglas LW, Veillon $\mathrm{C}$, Kelsay JL, et al. Sodium and potassium intake and balance in adults consuming self-selected diets. Am J Clin Nutr 1984; 40: 786-793.

23. Koopman MG, Koomen GC, Krediet RT, de Moor EA, Hoek FJ, Arisz L. Circadian rhythm of glomerular filtration rate in normal individuals. Clin Sci 1989; 77: 105-111.

24. Mann SJ, Gerber LM. Estimation of 24-hour sodium excretion from spot urine samples. J Clin Hypertens 2010; 12: 174-180.

25. Buemi M, Campo S, Sturiale A, Aloisi C, Romeo A, Nostro L, et al. Circadian rhythm of hydration in healthy subjects and uremic patients studied by bioelectrical impedance analysis. Nephron Physiol 2007; 106: 39-44.

26. Joossens JV, Kesteloot H. Trends in systolic blood pressure, 
24-hour sodium excretion, and stroke mortality in the elderly in Belgium. Am J Med 1991; 90: 5S-11S.

27. Staessen J, Broughton PM, Fletcher AE, Markowe HL, Marmot MG, Rose G, et al. The assessment of the relationship between blood pressure and sodium intake using whole-day, daytime and overnight urine collections. J Hypertens 1991; 9: 1035-1040.
28. Bankir L, Bochud M, Maillard M, Bovet P, Gabriel A, Burnier $M$. Nighttime blood pressure and nocturnal dipping are associated with daytime urinary sodium excretion in African subjects. Hypertension 2008; 51: 891-898.

29. Dyer A, Elliott P, Chee D, Stamler J. Urinary biochemical markers of dietary intake in the INTERSALT study. Am J Clin Nutr 1997; 65: 1246S-1253S. 